\title{
EEG activity during administration of low-concentration odors
}

\author{
TYLER S. LORIG, KATE B. HERMAN, and GARY E. SCHWARTZ \\ Yale University, New Haven, Connecticut \\ and \\ WILLIAM S. CAIN \\ John B. Pierce Foundation and Yale University, New Haven, Connecticut
}

\begin{abstract}
Previous research has suggested that room air may contain odors that affect central nervous system activity. This research presumes that odors can produce significant changes in brain activity even though they are undetected. In the present study, we examined this hypothesis. Sixteen subjects were exposed to four concentrations of two odorants while EEG data were recorded. The concentrations used in the study were no odor, low (undetected in half the subjects), medium, and high. The results of the EEG period analyses indicated that the distribution of EEG theta activity differed as a function of odor concentration. In addition, more detailed analysis of the two lowest odor-presentation conditions revealed that EEG beta activity differed as a function of odor and concentration for the subjects who were unable to detect the presence of the odors. These subjects also reported being significantly less happy during the administration of the undetected odors. These results support the hypothesis that undetected odors, which may exist in room air, have the capacity to alter brain activity and possibly mood.
\end{abstract}

Olfaction has often been considered to be a relatively unimportant sense in the direction of human behavior (Brodal, 1981). Human olfactory response is paradoxically characterized by extreme sensitivity to many odors and a limited ability to name them (Engen, 1987). Anatomically, the central nervous system (CNS) projections of the olfactory tract are widespread and reach much of the brain within two synapses of the olfactory bulb (Shepherd, 1979). Such a broad distribution of olfactory projections suggests that olfaction is important in the direction of functional brain processes and that olfactory stimuli should have a significant impact on brain activity. Recent evidence has indicated that odors can alter the distribution of EEG activity in humans (Lorig \& Schwartz, 1988), but the relatively large proportion of neural tissue associated with the olfactory tract and our limited use of odor information remains incongruous.

One possible explanation for this inconsistency is that olfaction may have its greatest impact on subcortical processes that operate outside awareness (i.e., in situations wherein observers claim that no odors are detectable). Like cortical blindness, a condition in which visual input affects behavior through subcortical tracts but awareness and subsequent description of the stimulus is lost (Hecean \& Albert, 1978), much of the olfactory system

Support for this research was provided by International Flavors and Fragrances. The authors wish to thank D. Elmes for his comments on the manuscript. G. E. Schwartz's current address is Department of Psychology, University of Arizona, Tucson, AZ 85721. Correspondence may be addressed to T. S. Lorig, who is currently at the Department of Psychology, Washington and Lee University, Lexington, VA 24450. may actively operate subcortically and in the absence of detection. It may be that only when attention is directed toward an odor, or an odor stimulus of great magnitude is encountered, are the channels to the cortex and awareness opened. Some evidence suggests that this gating of cortical input may take place in the thalamocortical pathway arising in the olfactory tubercule, since damage to this pathway spares detection but leads to an inability to describe odors (Castellucci, 1985).

Several lines of evidence support the hypothesis that odors can exert CNS effects outside awareness. McClintock (1971) has suggested that undetected odors may affect the regulation of the menstrual cycle. The hypothesis that olfaction can modulate the menstrual cycle has been supported by the work of Russell and colleagues (Russell, Switz, \& Thompson, 1980), but remains controversial. Lorig and Schwartz (1988) found that EEG activity was dissociated from the perception of detectable odors, and suggested that perceptual components of odors account for only a small proportion of their observed EEG effects. Control conditions using purified air samples have also been found to produce increased activity in the olfactory bulb when compared to resting levels, thus suggesting that even minor contaminants might affect CNS activity (Hughes, Hendrix, Wetzel, \& Johnson, 1969). In addition, several investigators have determined that inhalation of room air through the nose affects EEG (Lehman \& Knauss, 1976; Lorig, Schwartz, Herman, \& Lane, 1988; Werntz, Bickford, Bloom, \& Shannahoff-Khalsa, 1983). Although mentioned only by Lorig et al., these reports suggest the hypothesis that undetected odors significantly alter human brain activity. A thorough test of 
this hypothesis requires the direct manipulation of undetected odor conditions, however, and was the purpose of the present investigation.

\section{Method}

\section{Subjects}

Sixteen subjects ( 8 male and 8 female) responding to a posted advertisement participated in the experiment. The subjects ranged in age from 17 to 34 years, with a mean of 20.7 years. All subjects were right-handed nonsmokers who reported no history of head injury and had no colds or allergies at the time of the experiment.

\section{Materials and Procedure}

The subjects entered the laboratory, were acquainted with the procedures for the collection of EEG data, and signed informed consent forms. Gold-plated, cup-type (10-mm) electrodes were affixed to the subjects' scalps at Locations F7, F8, T5, and T6, using Elefix electrode paste. These positions were chosen because they represent the widest distribution of electrodes and have produced reliable differences in previous research (Lorig \& Schwartz, 1988; Lorig et al., 1988). Impedances were always below $10,000 \Omega$, and were typically below 5,000 $\Omega$. Linked mastoids served as a reference to the active electrode sites. EEG channels were amplified using Grass Instruments preamplifiers (Model 7P5a) and were digitized and stored on disk at a sampling rate of $128 \mathrm{~Hz}$ by an IBM-compatible computer, using a 12-bit Data Translation DT2801-A analog-to-digital converter. EEG data were collected within a $1-35-\mathrm{Hz}$ range and smoothed off-line to a $2-32-\mathrm{Hz}$ bandwidth. Eye-movement artifacts were eliminated by the period analysis algorithm, which ignored low-frequency segments of data that could have contained eye movements while quantifying the frequencies of primary interest in the present study (Lorig, 1986). Respiration and EKG data were also collected from several subjects but were not used in the analyses.

A constant-flow olfactometer (see Figure 1) provided for remote control of the concentration and duration of odor presentation. Odorants were presented to the subjects after being mixed with room air in the olfactometer. Odor chemicals were introduced silently into the air stream by means of metered valves and compressed breathing-grade air located outside the subject chamber. The gentle flow of air containing the odor mixture was directed toward the subject's face from a distance of $15 \mathrm{~cm}$. Thus, either room air or room air mixed with odorant constantly blew into the subject's face. Naglene tubing was used throughout the apparatus.

The subjects were instructed to sit quietly, with their eyes closed, and breathe normally during each period of data collection. They were told that odors might or might not be presented during any given trial. Two odorants, lavender oil and spiced apple (provided by International Flavors and Fragrances; Union Beach, NJ), were presented at four concentrations: none, low, medium, and high. These concentrations were based

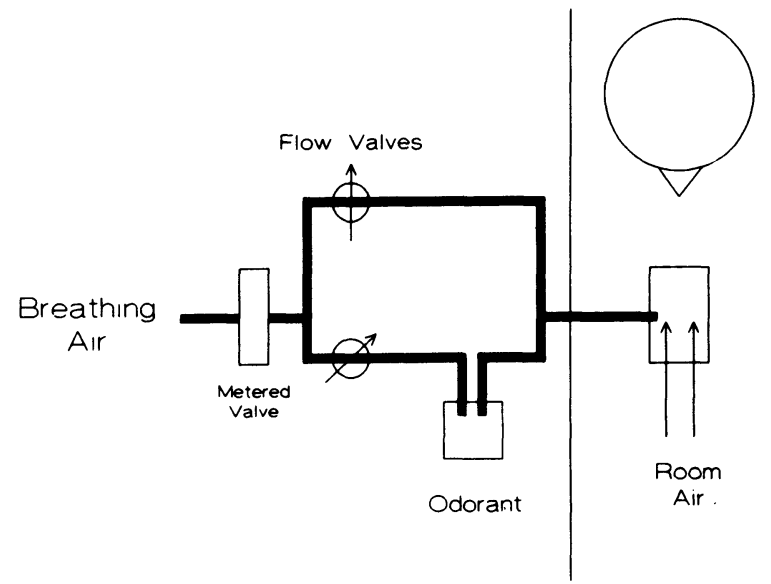

Figure 1. Constant-flow olfactometer. on preliminary pilot testing of a different group of subjects and were set so that the low-odor condition represented most subjects' odordetection thresholds, the medium condition represented a concentration detectable to all subjects, and the high condition represented a concentration of sufficient magnitude as to be identifiable or distinguishable from other odors. Odor-administration trials lasted $15 \mathrm{sec}$ and were given in counterbalanced order. EEG data were collected in one 15-sec epoch.

After each trial, the subjects were asked to complete a 17 -item questionnaire consisting of 10-point Likert scales associated with various terms such as "relaxed," "tense," "happy," and "bored," as well as questions on odor presence, intensity, and pleasantness. The room was ventilated during this time, and the interstimulus interval was approximately $3 \mathrm{~min}$.

Following completion of the experiment, the subjects were debriefed and paid.

\section{RESULTS AND DISCUSSION}

EEG data were reduced for the alpha $(8-13-\mathrm{Hz})$, beta $(13-64-\mathrm{Hz})$, and theta $(4-7-\mathrm{Hz})$ frequency ranges using period analysis. This form of analysis determines the duration or major period of each EEG wave and quantifies the number of EEG waves that occur in various frequency bands. This technique has been used in psychopharmacological (Fink, 1975) and task-performance studies (Harner, 1977) and in investigations of human odor response (Lorig \& Schwartz, 1988; Lorig et al., 1988). Comparisons of this technique with the more widely used spectral analysis have determined that period analysis is somewhat more sensitive for comparisons involving task performance, whereas spectral analysis is more sensitive to individual differences in EEG (Lorig, 1986). This may be the reason that some investigations of spectrally analyzed EEG in human odor response have produced equivocal findings (Hummel, Forster, Pauli, \& Kobal, 1989).

Each of the EEG frequency bands was analyzed using repeated measures analysis of variance (concentration $x$ odor $\times$ hemisphere $x$ anterior-posterior), which were corrected for sphericity. This approach was used rather than MANOVA because sensitivity is lost using MANOVA when the sample size is less than $k+20$ (Vasey \& Thayer, 1987). The results of the analysis of EEG alpha activity indicated a trend toward decreased alpha activity as a function of the four concentrations of odors $[F(3,45)=2.60$, Greenhouse-Geiser corrected $p=$ $.08]$. The analysis of theta activity indicated a significant change in the hemispheric and anterior-posterior distribution of theta activity as a function of concentration $[F(3,45)=3.37$, Greenhouse-Geiser corrected $p=.029]$. This difference was greatest between the low and medium odor-concentration conditions, and indicated more left posterior theta activity during administration of the low concentrations of both odors. Such differences may indicate an increase in cognitive activity associated with the medium odor concentration, since similar changes in the distribution of theta activity occur during some cognitive tasks, especially those requiring internal verbalization. Scalp topography maps of the distribution of theta activity are presented in Figure 2.

More important to the specific hypothesis under investigation was the determination of EEG changes that oc- 


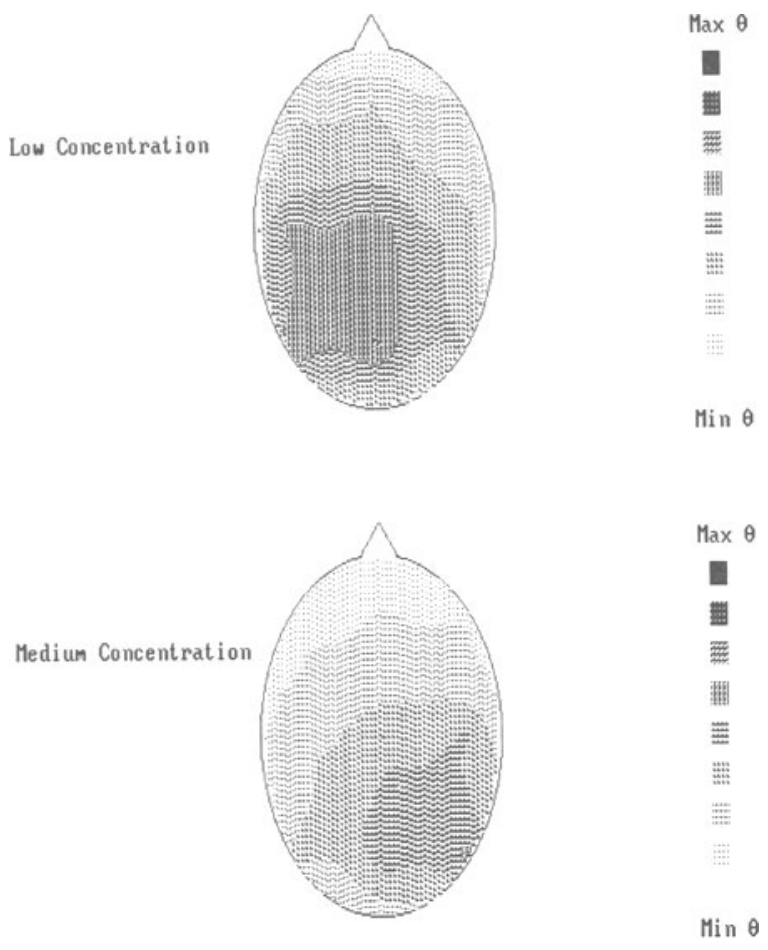

Figure 2. Scalp topography maps of theta activity for the low (top) and medium (bottom) odor conditions.

curred in subjects who did not smell the lowest concentrations of the two odors. For this reason, only the subjects who reported no detections of both the lavender and the spiced apple odors in the low-concentration condition were selected for further analysis. In addition, subjects who reported false detections of odor in both of the no-odor presentation conditions were excluded from analysis. This left a sample of 7 subjects who reported smelling nothing in both of these conditions. EEG data for these subjects were analyzed similarly to the analysis mentioned earlier (concentration $\times$ odor $\times$ hemisphere $\times$ anteriorposterior), except that only the no-odor and low-odor concentrations were included. Since two levels of each variable were used, Greenhouse-Geiser corrections were unnecessary. The results of these analyses indicated significant changes in beta activity $[F(1,6)=8.29, p=.028]$ and a tendency toward change in the theta range. The changes in beta activity indicated an alteration in the distribution of anterior and posterior activity that differed with the concentrations of the two odors. This relationship is presented in Figure 3. As is evident in the figure, the lavender odor produced a large increase in posterior beta activity relative to the frontal regions. This result is similar to previous findings (Lorig et al., 1988) in which nose inhalation of room air produced beta activity changes in the same anterior-posterior dimension. Increases in beta activity such as this are often due to the blocking of alpha activity, which is greatest across posterior regions of the head. Such changes may indicate cognitive activity, but are more commonly associated with cortical arousal due to sensory stimulation.

Analysis of the 17 items from the self-report data revealed only one item that differed as a function of concentration or odor. The subjects who did not detect the odors reported being less happy when the lowest (undetected) concentrations of either spiced apple or lavender odors were presented $[F(1,6)=6.08, p=.049]$. This effect is somewhat paradoxical and is difficult to interpret, given that the distribution of EEG activity for lavender and spiced apple changed in opposite directions and that previous research has indicated that suprathreshold administration of spiced apple produced increased self-reports of relaxation (Lorig \& Schwartz, 1988). The finding that undetected concentrations of spiced apple decreased selfreports of happiness may indicate that spiced apple's previous suprathreshold effects are due to cognitive mediation. This hypothesis is supported by data that indicate that imagery of food produces EEG changes similar to suprathreshold odor effects (Lorig \& Schwartz, in press).

Although previous investigations (Lorig \& Schwartz, 1988-89) have shown EEG theta differences due to different odorants, the findings reported here demonstrate that EEG theta activity also differs as a function of odor concentration. More importantly, these results indicate that even though some subjects did not report detection of the odor, odor-specific neurophysiological changes occurred. Furthermore, changes in mood (happiness) were reported when undetected concentrations were presented, but these results are difficult to interpret given the opposite shift in the distribution of cortical activity and self-reports for higher odor concentrations. The findings of this experiment strongly support the hypothesis that odors in low, undetected concentrations alter brain activity. However, the exact nature of this alteration remains unclear, since response to the low-concentration odors may indicate a simple arousal response typical of other sensory stimuli.

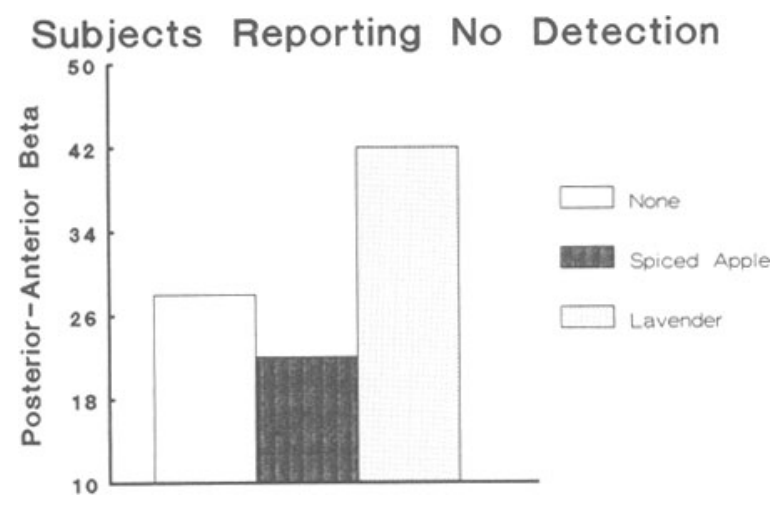

Figure 3. Anterior-posterior distribution of beta activity (posterior minus anterior) for subjects who did not detect the presence of odors in the no-odor and low-odor administration conditions. 


\section{REFERENCES}

Brodal, A. (1981). Neurological anatomy. New York: Oxford University Press.

CAstellucCI, V. F. (1985). The chemical sense: Taste and smell. In E. R. Kandel \& J. H. Schwartz (Eds.), Principles of neural science (2nd ed., pp. 409-425). New York: Elsevier.

EngEN, T. (1987). Remembering odors and their names. American Scientist, 75, 497-502.

FINK, M. (1975). Cerebral electrometry-quantitative EEG applied to human psychopharmacology. In G. Dolce \& H. Kunkel (Eds.), CEAN-Computerized EEG analysis (pp. 271-288). Stuttgart: Fischer.

HARNER, R. N. (1977). EEG analysis in the time domain. In A. Remond (Ed.), EEG informatics: A didactic review of methods and applications of EEG data processing (pp. 57-82). Amsterdam: Elsevier.

HeCEAN, H., \& AlBerT, M. L. (1978). Human neuropsychology. New York: Wiley.

Hughes, J. R., Hendrix, D. E., Wetzel, N., \& Johnson, J. W., JR. (1969). Correlation between electrophysiological activity from the human olfactory bulb and the subjective response to odoriferous stimuli. In C. Pfaffmann (Ed.), Olfaction and taste (pp. 000-000). New York: Rockefeller University Press.

Hummel, T., Forster, S., Pauli, E., \& Kobal, G. (1989, April). Are there correlates of hedonic estimates in the power spectra of the human EEG? Paper presented at the meeting of the Association for Chemoreception Sciences, Sarasota, FL.

Lehmann, D., \& KNauss, T. A. (1976). Respiratory cycle and EEG in man and cat. Electroencephalography \& Clinical Neurophysiology, 40, 187.
LORIG, T. S. (1986). EEG and task performance: A comparison of three analytic techniques. Physiological Psychology, 14, 130-132.

Lorig, T. S., \& SchwarTz, G. E. (1988). Brain and odor: I. Alteration of human EEG by odor administration. Psychobiology, 16, 281-284.

LORIG, T. S., \& SCHWARTz, G. E. (1988-89). EEG activity during food and relaxation imagery. Imagination, Cognition \& Personality, 8, 201-208.

Lorig, T. S., Schwartz, G. E., Herman, K. B., \& Lane, R. D. (1988). Brain and odor: II. EEG activity during nose and mouth breathing. Psychobiology, 16, 285-287.

MCClintocK, M. K. (1971). Menstrual synchrony and suppression. Nature, 229, 244-245.

Russell, M. J., Switz, G. M., \& Thompson, K. (1980). Olfactory influences on the human menstrual cycle. Pharmacology, Biochemistry \& Behavior, 13, 737-738.

SHEPHERD, G. M. (1979). The synaptic organization of the brain (2nd ed.). New York: Oxford University Press.

VASEY, M. W., \& ThAYER, J. F. (1987). The continuing problem of false positives in repeated measures ANOVA in psychophysiology: A multivariate solution. Psychophysiology, 24, 479-486.

Werntz, D. A., Bickford, R. G., Bloom, F. E., \& ShannahoffKHALSA, D. S. (1983). Alternating cerebral hemisphere activity and the lateralization of autonomic nervous function. Human Neurobiology, 2, 39-43.

(Manuscript received January 23, 1990.) 